\title{
Vector distribution and transmission risk of the Zika virus in South and Central America
}

\author{
Sarah Cunze ${ }^{\text {Corresp., } 1}$, Judith Kochmann ${ }^{1}$, Lisa K. Koch ${ }^{1}$, Elisa Genthner $^{1}$, Sven Klimpel ${ }^{1}$ \\ ${ }^{1}$ Goethe University, Institute of Ecology, Evolution and Diversity; Senckenberg Biodiversity and Climate Research Centre, Frankfurt am Main, Germany \\ Corresponding Author: Sarah Cunze \\ Email address: cunze@bio.uni-frankfurt.de
}

Background: Zika is of great medical relevance due to its rapid geographical spread in 2015 and 2016 in South America and its serious implications, e.g. certain birth defects. Recent epidemics urgently require a better understanding of geographic patterns of the Zika virus transmission risk. This study aims to map the Zika virus transmission risk in South and Central America. We applied the maximum entropy approach, which is common for species distribution modelling, but is now also widely in use for estimating the geographical distribution of infection diseases. Methods: As predictor variables we used a set of variables considered to be potential drivers of both direct and indirect effects on the emergence of Zika. Specifically, we considered (a) the modelled habitat suitability for the two main vector species Aedes aegypti and Aedes albopictus as a proxy of vector species distributions; (b) temperature, as it has a great influence on virus transmission; (c) commonly called evidence consensus maps of human Zika virus infections on a regional scale as a proxy for virus distribution; (d) evidence consensus maps of human dengue virus infections and, (e) as possibly relevant socio-economic factors, population density and the gross domestic product. Results: The highest values for the Zika transmission risk were modelled for the eastern coast of Brazil as well as in Central America, moderate values for the Amazon basin and low values for southern parts of South America. The following countries were modelled to be particularly affected: Brazil, Colombia, Cuba, Dominican Republic, El Salvador, Guatemala, Haiti, Honduras, Jamaica, Mexico, Puerto Rico and Venezuela. While modelled vector habitat suitability as predictor variables showed the highest contribution to the transmission risk model, temperature of the warmest quarter contributed only comparatively little. Areas with optimal temperature conditions for virus transmission overlapped only little with areas of suitable habitat conditions for the two main vector species. Instead, areas with the highest transmission risk were characterised as areas with temperatures below the optimum of the virus, but high habitat suitability modelled for the two main vector species. Conclusion: Modelling approaches can help estimating the spatial and temporal dynamics of a disease. We focused on the key drivers Peerj reviewing PDF | (2019:04:37005:2:0:NEW 27 Aug 2019) 
relevant in the Zika transmission cycle (vector, pathogen, and hosts) and integrated each single component into the model. Despite the uncertainties generally associated with modelling, the approach applied in this study can be used as a tool and assist decision making and managing the spread of Zika. 
1 Vector distribution and transmission risk of the Zika virus in South and Central

2 America

3 Sarah Cunze $^{1}$, Judith Kochmann ${ }^{1}$, Lisa K. Koch ${ }^{1}$, Elisa Genthner ${ }^{1}$, Sven Klimpel ${ }^{1}$

$4{ }^{1}$ Goethe University, Institute of Ecology, Evolution and Diversity; Senckenberg Biodiversity and

5 Climate Research Centre, Frankfurt am Main, Germany

6

7 sarahcunze@gmail.com

8 Judith.Kochmann@senckenberg.de

9 L.Koch@bio.uni-frankfurt.de

10 elisa_genthner@yahoo.de

11 Klimpel@bio.uni-frankfurt.de

12

*Corresponding author: Sarah Cunze, Goethe-University, Institute for Ecology, Evolution and

Diversity; Max-von-Laue-Str. 13, D-60438 Frankfurt/ M., Germany, Phone: +49 (0)69 798

42245, Fax: +49 (0)69 798 42236, sarahcunze@gmail.com 
16

17

18

19

20

Abstract

Background: Zika is of great medical relevance due to its rapid geographical spread in 2015 and 2016 in South America and its serious implications, e.g. certain birth defects. Recent epidemics urgently require a better understanding of geographic patterns of the Zika virus transmission risk. This study aims to map the Zika virus transmission risk in South and Central America. We applied the maximum entropy approach, which is common for species distribution modelling, but is now also widely in use for estimating the geographical distribution of infection diseases.

Methods: As predictor variables, we used a set of variables considered to be potential drivers of both direct and indirect effects on the emergence of Zika. Specifically, we considered (a) the modelled habitat suitability for the two main vector species Aedes aegypti and Aedes albopictus as a proxy of vector species distributions; (b) temperature, as it has a great influence on virus transmission; (c) commonly called evidence consensus maps of human Zika virus infections on a regional scale as a proxy for virus distribution; (d) evidence consensus maps of human dengue virus infections and, (e) as possibly relevant socio-economic factors, population density and the gross domestic product.

Results: The highest values for the Zika transmission risk were modelled for the eastern coast of Brazil as well as in Central America, moderate values for the Amazon basin and low values for southern parts of South America. The following countries were modelled to be particularly affected: Brazil, Colombia, Cuba, Dominican Republic, El Salvador, Guatemala, Haiti, Honduras, Jamaica, Mexico, Puerto Rico and Venezuela.

While modelled vector habitat suitability as predictor variables showed the highest contribution to the transmission risk model, temperature of the warmest quarter contributed only comparatively 
38 little. Areas with optimal temperature conditions for virus transmission overlapped only little with

39 areas of suitable habitat conditions for the two main vector species. Instead, areas with the highest

40 transmission risk were characterised as areas with temperatures below the optimum of the virus,

41 but high habitat suitability modelled for the two main vector species.

42 Conclusion: Modelling approaches can help estimating the spatial and temporal dynamics of a

43 disease. We focused on the key drivers relevant in the Zika transmission cycle (vector, pathogen,

44 and hosts) and integrated each single component into the model. Despite the uncertainties

45 generally associated with modelling, the approach applied in this study can be used as a tool and

46 assist decision-making and managing the spread of Zika. 
48

49

50

51

52

53

54

55

56

57

58

59

60

61

62

63

64

65

66

67

68

69

70

\section{Introduction}

Vector-borne diseases are globally emerging or re-emerging as a consequence of global warming, globalization in trade and travel, changes in modern transport networks, and urbanization (Medlock \& Leach 2015; Balogun, Nok \& Kita, 2016). The arthropod-borne Zika flavivirus (ZIKV) is of great medical importance as it has the potential to cause serious complications in a small proportion of infected individuals (WHO 2016; Krauer et al., 2017). Recent epidemics of Zika disease (or Zika fever, or Zika) in South America in 2015 and 2016 have raised awareness of an existing need for a better understanding of the geographical patterns of the ZIKV transmission risk.

Zika has not been recognized as an important disease in humans for a long time after its first discovery in 1947 in Uganda (Županc \& Petrovec 2016), probably due to previous outbreaks being limited in size and poor birth defect surveillance in areas where it is transmitted. Only a small proportion of cases of ZIKV infections are symptomatic (Funk et al., 2016). For a long time, symptomatic ZIKV infections were only reported from few small areas in Africa and Asia, and ZIKV was thus considered to be of limited public health importance until 2007 (Lessler et al., 2016; Messina et al., 2016). In 2014, ZIKV was found in the Americas for the first time, and it rapidly spread throughout several South American states. Following a considerable increase in the incidence of microcephaly in newborns, especially during an outbreak in Brazil in 2015, and of Guillain-Barré syndrome, alarms were raised worldwide (Lessler et al., 2016). In response to these potentially severe complications and the rapid expansion of ZIKV into previously unaffected areas, as well as the size of the outbreak, on 1 February 2016 the World Health Organization (WHO) declared a public health emergency of international concern (Županc \& Petrovec 2016; Lessler et al., 2016). Despite declining numbers of Zika infections throughout 2017 and 2018, the Pan 
71 American Health Organization (PAHO) maintains reports of cases of ZIKV infection and 72 congenital Zika syndrome from the Americas (WHO, 2019).

73 Mapping the geographical distribution of diseases and identifying potential areas under risk of 74 infections is crucial for evidence-based decision making in public health (e.g. Escobar 2017). 75 Several modelling attempts have recently been made for Zika based on correlative (e.g. Samy et al., 2016, Messina et al., 2016, Lo et al., 2018) and mechanistic approaches (e.g. Funk et al., 2016, Suparit et al., 2018). Applications of correlative ENM approaches focused on modelling the potential distribution of single 'components' (cf. Johnson et al. 2019) of the transmission cycle (e.g. Baak-Baak et al., 2017 for vector species and Samy et al., 2017 for vector species and parasites), such as pathogens (bacteria, viruses, or parasites), vectors, and hosts (Escobar \& Craft 2016, Johnson et al., 2019). Other approaches directly linked disease occurrences to environmental factors (e.g. Pigott et al. 2015), which is especially useful when information on transmission mechanisms or distributional data of vectors or pathogens is missing. Given the large number of important factors affecting the temporal and spatial patterns of a disease and which cannot be adequately mathematically described in their complexity, the integration of empirical data in correlative approaches seems to be the most feasible way to map the geographical distribution of diseases (cf. Messina et al. 2015).

Here, we chose a correlative ENM approach to estimate the ZIKV transmission risk in South and Central America. Our approach is a mix between the two aforementioned approaches, accounting for the key components of the transmission cycle (e.g. modelled habitat suitability for vector species, ECM of virus distribution) and linking those to occurrences of disease cases. Reliable estimations in ENM should account for all relevant factors shaping the potential distribution of the disease. A major component of the ZIKV transmission cycle are the vector species and their 
94 distribution. ZIKV is transmitted by mosquitoes, with Aedes aegypti and Ae. albopictus being the

95 primary and secondary vectors (Heitmann et al., 2017). Hence, when modelling the geographical

96 distribution of ZIKV transmission risk, the distribution of these two vectors needs to be

97 established. In our approach, we first estimated the climatic habitat suitability for the two main

98 vector species (Ae. albopictus and Ae. aegypti) to generate a proxy for the vector species'

99 distribution. Secondly, we used regional information on disease incidences as a proxy for the

100 presence of the virus itself. This information was based on databases of several health

101 organizations (i.e. World Health Organization - WHO; Centers for Disease Control and Prevention

102 - CDC; Global Infectious Disease and Epidemiology Network - Gideon) and accounted for by

103 creating a commonly called evidence consensus map (ECM). In addition to the distributions of

104 these key actors (vector species and virus), abiotic factors such as temperature also play an

105 important role. We included mean temperature of the warmest quarter, as temperature is

106 considered an important driver of the virus transmission risk for vector borne diseases (Mordecai

107 et al., 2017; Tesla et al., 2018). We further considered population density and gross domestic

108 product to include socio-economic factors, potentially related to the risk of infection (cf. Romeo-

109 Aznar et al., 2018).

110 Our aim was to describe a general pattern and test the relevance of each predictor based on

111 currently, easily accessed information using ENM as a tool. The approach applied in this study

112 might be used as a tool and assist decision-making and managing the spread of Zika. 


\section{Material and Methods}

115 We used a correlative approach to estimate the ZIKV transmission risk in South and Central 116 America following the approach suggested in (Messina et al., 2015). The graphical abstract given

117 in Fig. 1 refers to the data used in our modified approach. The study area covers 27 countries in 118 Central and South America.

119 The final model of the ZIKV transmission risk is based on known occurrences of the disease taken 120 from (Messina et al., 2016; Messina \& Shearer 2016, $\mathrm{N}=239$ ) and the following predictor 121 variables: a) habitat suitability maps of the two main vector species, Ae. albopictus and Ae. aegypti 122 (as a proxy for vector species' distribution, see below for further details), b) temperature of the 123 warmest quarter, c) evidence consensus map for Zika (cf. Brady et al., 2012), d) evidence 124 consensus map for dengue (with the same vector species as Zika), e) population density, and f) 125 gross domestic product per capita (on country level).

\section{ENM for vector species}

127 As species distribution is mainly driven by climatic conditions on a continental scale, we 128 considered 19 bioclimatic variables provided by worldclim (version 2.0, www.worldclim.org, Fick

129 \& Hijmans 2017) to model the habitat suitability for the two main vector species. In addition to 130 climatic conditions, land cover is considered to be an important driver shaping species' 131 distributional patterns. Land cover variables were based on data from the "GlobeCover 2009 land 132 cover map" (Arino et al., 2012) provided by the ESA (European Space Agency, ESA 2010). 133 According to the United Nations Land Cover Classification Systems (LCCS) land cover is 134 classified into classes, out of which we chose 20 classes for the considered study area (see Table 135 S2). Based on the spatial resolution of the climatic data used, we calculated the percentage of each 
136 land cover class per grid cell, resulting in 20 variable layers with the percentages of the respective

137 land cover class ranging from $\quad 0 \%$ to $\quad 100 \%$.

139 To reduce the dimensionality of predictor variables, we have applied two approaches. First, we

140 chose 10 out of the 39 original variables that we consider ecologically relevant (five climatic and

141 five landcover). Secondly, we performed a principal component analysis (PCA) for the 39

142 variables (i.e. 19 bioclimatic variables and 20 land cover variables) using the $\mathrm{R}$ function

143 "rasterPCA" implemented in the package "RStoolbox" version 0.1.10 (Leutner \& Horning 2017).

144 For further analysis, we used the ENM results with the comparably highest area under the receiver 145 operating characteristic curve (AUC) value.

146 To model the habitat suitability of the two main vector species Ae. aegypti and Ae. albopictus as

147 well as the ZIVK transmission risk, we chose the maximum entropy approach implemented in the

148 Maxent software (version 3.4.1) (Phillips et al., 2017), which is a presence-background approach

149 and particularly suitable when reliable absence data is missing.

150 Occurrence data for both species were taken from Kraemer et al. (2015a,b, N = 6716 occurrences

151 for Ae. aegypti and $\mathrm{N}=3526$ occurrences for Ae. albopictus ). Occurrence records were adjusted

152 to the spatial resolution of the environmental data ( 5 arc minutes $* 5$ arc minutes) and only one

153 record per grid cell was used for ENM analysis in order to reduce spatial biases (see e.g. Aiello-

154 Lammens et al., 2015) resulting in $\mathrm{N}=5150$ occurrences for Ae. aegypti and $\mathrm{N}=3303$ occurrences

155 for Ae. albopictus.

156 As predictor variables we used the first nine principal components derived from the above

157 mentioned PCA (with corresponding eigenvalues greater than one; Kaiser criterion), explaining 
$15866.9 \%$ of the variability of the original data set of 39 variables (Fig. 1). We thus accounted for

159 inter-correlation and were able to reduce the number of variables incorporated in the ENM with

160 the least possible loss of information.

161 Two habitat suitability maps with continuous values between 0 (no habitat suitability) and 1 (full

162 habitat suitability) for Ae. aegypti and Ae. albopictus were created.

\section{ENM for the ZIKV transmission risk}

To model the ZIKV transmission risk we accounted for the main key drivers: vector distribution, virus distribution, temperature and socio-economic factors. Specifically we used the following seven predictor variables: the modelled habitat suitability for Ae. albopictus and Ae. aegypti (see previous section), mean temperature of the warmest quarter (bio10) as provided by worldclim, the evidence consensus maps for Zika and dengue, as well as population density and gross domestic product (see below for further details).

The temperature of the warmest quarter was chosen as abiotic variable due to the strong impact of temperature on virus transmission (Mordecai et al., 2017; Tesla et al., 2018). Detailed descriptions of the other variables are following below.

\section{ECM for Zika}

The method of ECM, (see Brady et al., 2012) has been developed to better compile disparate data types and synthesize information in a robust and repeatable way. It generally summarizes information for a country or other administrative units and displays it in a map. 
179 We compiled and standardised information on reported Zika cases between 2015 and 2017 from

180 various sources, specifically from the World Health Organization - WHO

181 (http://www.who.int/en/); Centres for Disease Control and Prevention - CDC

182 (https://www.cdc.gov/); Global Infectious Disease and Epidemiology Network - Gideon

183 (https://www.gideononline.com/), Pan American Health Organization - PAHO

184 (http://www.paho.org/hq/). The considered administrative units were regions, departments, 185 provinces and states (depending on the country). The geometries of these areas were taken from 186 the esri ArcGIS online database.

187 With reference to Brady et al., 2012 we first calculated the evidence consensus value for the Zika 188 transmission risk. These were based on country level records obtained by the above mentioned 189 sources, ranging from 0 (absence) to 12 (highest presence) according to the scoring system 190 displayed in Fig. 2. In a second step, this information were refined, using more regional data on 191 incidence rates or case numbers (depending on availability). For each administrative unit (region, 192 department, etc.) the country level value is scaled by an administrative-unit-specific value between 1930 and 1 referring to the relative incidence rate or case number in this region.

\section{ECM for dengue}

196

197

198

Infections of ZIKV together with infections of DENV are known from various studies (DupontRouzeyrol et al., 2015) and it has been suggested that the risk of symptomatic Zika is higher in people who have had prior dengue infections (Dejnirattisai et al., 2016; Cohen 2017). We thus used the dengue ECM generated by Brady et al., (2012), which ranges from complete absence to good absence, moderate absence, poor absence, intermediate, poor presence, moderate presence, 
201 good presence and complete presence. We transformed these nine classes to numbers from 0 to 8 ,

202 with only five different categories occurring in the study area ( 0 complete absence, 2 moderate

203 absence, 6 moderate presence, 7 good presence, 8 complete presence).

Population density and gross domestic product

206

207

208

209

210

211

212

213

214

215

216

217

218

219

220

221

Additionally, we considered the population density (population number on a country scale was taken from Gideon; information on area was taken from https:/www.worldatlas.com) and the gross domestic product (GDP) per capita (World Bank, 2016) on a country level as predictor variables.

\section{Further Analyses}

Among the seven predictor variables for the ZIKV transmission risk, the evidence consensus for Zika as well as for dengue were considered as categorical variables. The other five variables (habitat suitability for Ae. aegypti, habitat suitability for Ae. albopictus, mean temperature of the warmest quarter, population density, gross domestic product) were considered as continuous variables. We displayed the one-variable response curves built with Maxent to assess whether the relationships between the modelled ZIKV transmission risk and any of the predictor variables were as expected. We assumed the following relationships: a) the higher the habitat suitability for the vector species, the higher the ZIKV transmission risk b), the higher the temperature, up to a certain

threshold, the higher the ZIKV transmission risk, c) the higher the incidence rates of the Zika (accounted for by the evidence consensus values for Zika) the higher the ZIKV transmission risk, 
222 d) the higher the incidence rates of dengue, the higher the incidence rates of Zika (as both diseases

223 share the same vector species) e) the higher the population density, the higher the ZIKV

224 transmission risk and f) the higher the gross domestic product per capita, the lower the ZIKV

225 transmission risk.

226 A jack-knifing test for variable importance is implemented in the Maxent software and was 227 undertaken to analyse training gains.

\section{Software}

229 The maximum entropy modelling approach implemented in the software Maxent (version 3.4.1, 230 Phillips et al. 2017) was used for modelling the habitat suitabilities of the two vectors as well as 231 for the ZIKV transmission risk. We used the default settings in Maxent but only used linear, 232 quadratic and product features, and excluded hinge features (cf. Cunze \& Tackenberg, 2015). The 233 restriction to linear, quadratic and product features yields smooth response curves (continuous and 234 differentiable response curves). In addition, we enhanced the maximum number of iterations to 23550000 to ensure convergence. Maxent is an algorithm that is often used and performs well in model comparison (e.g. Elith et al., 2006). As a "presence-background model", Maxent is especially

237 suitable for studies without reliable absence data.

238 Further analysis was performed in R (R Core Team, 2014) and ESRI ArcGIS (Release 10.3) and 239 the latter one was also used building the maps. 


\section{Results}

242 Ecologically relevant variables were chosen based on higher AUC values, thus, we chose the PCA

243 approach over the approach using ten variables for further analysis. For Aedes aegypti the AUC

244 values for the ENMs based on nine PCA components was $\mathrm{AUC}=0.788$ and thus slightly higher

245 than the AUC value of 0.732 for an ENM based on five land cover variables and five climatic

246 variables. For Aedes albopictus the AUC values for the ENMs based on nine PCA components

247 was $\mathrm{AUC}=0.867$, again slightly higher than the AUC value of 0.803 for an ENM based on five

248 land cover variables and five climatic variables.

249 The patterns of modelled habitat suitability for both vector species is in good accordance with the

250 patterns of their observed occurrence data $(\mathrm{AUC}=0.788$ for Aedes aegypti and $\mathrm{AUC}=0.867$ for

251 Aedes albopictus; Fig. 3). Both vector species show a similar distribution, with Ae. aegypti

252 showing also occurrences beyond the main distribution of Ae. albopictus (Fig. 3a). This could be

253 related to the fact that this species has been spreading in South America for a longer time than $A e$.

254 albopictus and accordingly.

255 We provide an ECM (sensu Brady et al., 2012) for Zika in South and Central America on a more 256 regional scale (Fig. 4).

257 For southern parts of South America as well as the Amazon basin only very low ZIKV transmission 258 risks ("nearly absent" or "low") were modelled (AUC $=0.860$ for the ZIKA transmission risk

259 model; Fig. 5), whereas large parts of Brazil (especially the east of Brazil), Colombia and 260 Venezuela, Mexico and Central America as well as the Caribbean were modelled to be at a "very 261 high" risk. The following countries are modelled to be particularly affected: Brazil, Colombia, 
262 Cuba, Dominican Republic, El Salvador, Guatemala, Haiti, Honduras, Jamaica, Mexico, Puerto 263 Rico and Venezuela.

264 According to the one-variable response curves (Fig. S1) there are positive relations between the 265 modelled ZIKV transmission risk in South and Central America (cloglog Maxent output) and: a)

266 the modelled habitat suitability for Ae. aegypti - the main vector species for Zika in South and 267 Central America (i.e. the higher the modelled habitat suitability for Ae. aegypti, the higher the 268 modelled ZIKV transmission risk), b) the modelled habitat suitability for Ae. albopictus - the 269 second main vector species for Zika in South and Central America (here not monotonic but with an optimum at rather high modelled habitat suitabilities for the vector species, c) the mean temperature of the warmest quarter (again not monotonic but with an optimum at high temperatures), d) the evidence consensus classes (categorical variable) for Zika and dengue, and e) the population density. On the other hand, the modelled ZIKV transmission risk (cloglog Maxent output) and the gross domestic product have a monotonic negative relationship. isolation (dark blue bars), for the model leaving out the respective variable but considering all 277 other variables (green bars), or for the regularized training gain for the model with all seven 278 variables (red bar).

The environmental variable with the highest gain in the jack-knifing (Fig. S2) when used in isolation is the modelled habitat suitability for Ae. aegypti, the main vector for ZIKV transmission. The environmental variable that decreases the model's gain the most when omitted is the Zika 282 evidence consensus class. These classes therefore appear to have most of the information not present in the other variables. Population density and gross domestic product showed the lowest 
284 contribution to the model according to the jack-knifing, with the lowest gain if used in isolation 285 and the lowest effect on the model gain when left out. 


\section{Discussion}

288 The spatial and temporal patterns of vector-borne diseases are driven by a high number of different

289 environmental, ecological and socio-economic factors with unknown influence and interaction

290 strength (Lessler et al., 2016), challenging predictive models on the ZIKV transmission risk.

291 Recently, there have been several attempts to model the ZIKV transmission risk on a global

292 (Messina et al., 2016; Samy et al., 2016; Carlson, Dougherty \& Getz W 2016; Caminade et al., 293 2017) or more local scale (e.g. Wiwanitkit \& Wiwanitkit, 2016 for Thailand, Lo \& Park, 2018 for

294 Brazil), however, the spatial and temporal patterns of ZIKV transmission are still not very well 295 understood.

296 Ecological niche modelling has become one of the most widely used approaches in estimating the 297 geographical distribution of emerging infection diseases (Escobar \& Craft, 2016). Here, it was 298 used to gain a better understanding of the recent spread of ZIKV in the tropical countries of South and Central America and to identify areas with a high ZIKV transmission risk. We accounted for key drivers in the transmission cycle of vector-borne disease (i.e. vector, pathogens, and hosts) and used a set of variables, which are considered to be potential drivers of both direct and indirect 302 effects on the emergence of Zika.

Generally, the map of the modelled ZIKV transmission risk is in large accordance with the occurrence of the mosquitoes, i.e. the ZIKV transmission risk seems to be highest in the eastern coast of Brazil as well as in Central America. The relation between the single predictor variables and the modelled ZIKV transmission risk as response variable shown in the response curves matches our expectations. Results of the jack-knifing identified the vector species distribution (habitat suitability maps for Ae. aegypti and Ae. albopictus) and the Zika distribution (Zika ECM) 
309 as the most important predictor variables (as expected), whereas the population density and gross

310 domestic product with a very coarse spatial resolution on country scale were of minor relevance

311 for the model. In the following paragraphs we discuss the explanatory variables used in the

312 modelling approach in more detail.

313 The primary source of ZIKV infection in humans is from bites of infected mosquitoes (Lessler et

314 al., 2016). We therefore took the modelled habitat suitability for the primary and secondary vectors

315 of ZIKV transmission, Ae. aegypti and Ae. albopictus (Heitmann et al., 2017), respectively as a

316 proxy for vector distribution. Both species show similar distribution patterns (based on the

317 recorded occurrences), leading to potential competitive exclusion in certain regions (Lounibos \&

318 Juliano 2018). However, in our case this does not contradict the general approach, since both

319 species are vector-competent. Both vector species show their main distribution along the eastern

320 coast of Brazil. This area is as a hotspot of occurrence records and consequently shows a high

321 projected habitat suitability modelled for both species.

322 When modelling the habitat suitability for the two vector species, we accounted for climatic factors

323 as well as land cover as important drivers shaping their potential distribution. The PCA was

324 implemented to reduce the dimensionality of predictor variables (Anderson \& Gonzalez, 2011;

325 Radosavljevic \& Anderson, 2014) although this implicates that modelling results are more difficult

326 to interpret ecologically. An alternative way would have been to only choose environmental

327 variables supposed to be ecologically relevant to the species at hand (e.g. Anderson, 2013). In fact,

328 we ran two different models for each vector species (one based on the principle components and

329 one based on five climatic and five land cover variables considered to be relevant). Both

330 approaches yielded similar patterns of the modelled habitat suitability of the vector species. For 
331 the further analyses we decided to take the models showing the higher AUC values, i.e. the ENM

332 models based on nine PCA components for both vector species.

333 As expected, we found a positive relationship (monotonously increasing response curve) between

334 the modelled ZIKV transmission risk and the modelled habitat suitability for both vector species.

335 The modelled habitat suitability for both vector species is highly important for the model, with

336 high gain values (i.e. a quality measure for modelling, the higher the gain, the better the model)

337 using either of the two as the only variable for the model, and a high loss in explanatory

338 gain/importance when leaving the variable out, especially for Ae. aegypti. The lower effects when

339 omitting the habitat suitability for Ae. albopictus from the model can be explained by the high

340 explanatory power of the other, ecologically similar vector species still present in the model, Ae.

341 aegypti.

342 In addition to the distribution of a disease vector, the distribution of the virus and its prevalence

343 are also very important factors when assessing the risk of getting infected. As an estimator for this

344 factor, we incorporated the commonly called evidence consensus map. We provided a Zika ECM

345 based on available information with a higher spatial resolution than only country level, which to

346 our knowledge is something that has not been attempted before. However, the resolution still

347 remains quite low. This is reflected in the final risk map and becomes apparent in discontinuities

348 in the modelled ZIKV transmission risk, e.g. following regional boundaries, especially in

349 Argentina and its neighbouring countries. Our approach for the regional ECM might underestimate

350 the occurrence of the disease for some regions. For example, for the state of Pernambucco (north-

351 eastern Brazil), our regional ECM gives a relatively low EC value of 1.6. Although this value

352 refers to 896 reported possible cases of Zika (de Brito et al., 2016), the prevalence of Zika in this

353 region is clearly lower than in other Brazilian states according to the PAHO case reports, 
354 (http://www.paho.org/data). The general patterns are well reflected by our map. The distribution

355 and prevalence of the virus are very important factors when assessing the risk of getting infected.

356 Our Zika ECM based on incidences of Zika infections is one of the best available estimators for

357 the prevalence of the virus (provided as Ascii-file in Supplementaries).

358 We used the dengue ECM as predictor variable assuming a positive correlation between the occurrence of DENV infections and the occurrence of ZIKV infections as both viruses share the same vector species. At the same time, there is some evidence that dengue immunity can be protective against Zika infection (Gordon et al. 2019, Rodriguez-Barraquer et al. 2019). In the jack-knifing, the Zika ECM clearly shows a higher variable contribution compared to the dengue ECM, with the highest loss in training gain when leaving the respective variable out. Both variables were taken as categorical variables, resulting in discontinuous response curves.

Temperature is considered a very important driver of vector-borne disease transmission due to temperature conditions affecting the life history of the vector species (Brand \& Keeling, 2017). Mosquitoes as ectothermic insects are especially known to be temperature-sensitive. Transmission can only succeed if the mosquito is able to survive the extrinsic incubation period between becoming infectious and biting new hosts (Tesla et al., 2018). In addition, the transmissibility of vector-borne viruses is also supposed to be affected by temperature (Samuel et al., 2016) as temperature conditions affect pathogen growth and survival in vector organisms (Brand \& Keeling, 2017). It has also been suggested that temperature has an indirect effect on the vector competence

373 of Aedes aegypti and Ae. albopictus for dengue virus transmissions through temperature dependence of Wolbachia infections (Tsai et al., 2017). To account for this assumed temperature dependency, we considered temperature in the transmission risk model, taking the temperature of 376 the warmest quarter (bio10) provided by worldclim as a separate predictor variable. Virus 
377 transmission (Zika, dengue, and chikungunya) has been suggested to occur between $18-34^{\circ} \mathrm{C}$ with

378 a maximum transmission rate from $26-29^{\circ} \mathrm{C}$ (Mordecai et al., 2017). The shape of the one-variable

379 response curve for temperature dependency of the modelled transmission risk matches this

380 suggested range very closely. We also compared areas with modelled habitat suitability for at least

381 one of the two main vector species (in red) and the area matching the temperature criteria as

382 suggested by Mordecai et al. (2017) (Figure S4). According to these results, habitat suitability for

383 the vector species and the optimal temperature conditions for ZIKV only match in very few areas,

384 whereas there are wide overlaps with the broader temperature range for the ZIKV and the modelled

385 habitat suitability for the mosquitoes. In areas with higher temperatures, i.e. $26-29^{\circ} \mathrm{C}$ (Fig. S4),

386 regarded as transmission optimum according to Mordecai et al. (2017), the modelled habitat

387 suitability for the two vector species is comparatively low (Fig. 3). There are also few occurrence

388 points for the disease (Fig. 1). The transmission risk is thus modelled to be highest in areas with

389 temperatures below the optimum of the virus, but within the range indicated by Mordecai et al.

390 (2017) and where the modelled habitat suitability for the two main vector species is high. While

391 the modelled habitat suitability as a predictor variable shows the highest contribution to the model

392 (Fig. S2), the temperature of warmest quarter only shows a comparatively small contribution .

393 As expected, the modelled ZIKV transmission risk is positively related to population density.

394 Socio-economic factors, such as urban poverty and overcrowding, and poor public health

395 infrastructure (cf. Bhatt et al., 2013 considering dengue) have been suggested to impact the

396 dynamics of ZIKV transmission. The gross domestic product per capita (GDP) was therefore

397 included as an indirect measure of health care provision. Gardner et al. (2018) found regional low

398 GDP to be the best predictor of ZIKV transmission, suggesting that Zika is primarily a disease

399 related to poverty. However, this variable scored only a very low variable importance in the jack- 
400 knifing test, which might be explained, at least partially, by a very low spatial resolution (i.e. on

401 country level) of population density and gross domestic product.

402 Disease biogeography is a very complex field, challenging the development of reliable models for

403 mapping disease risk. All models are therefore subject to a number of uncertainties and limitations.

404 Crucial for the performance and reliability of models is the quality of input data, which are often

405 biased as the reporting rate and geographical precision of occurrence data for diseases may vary

406 greatly by country (Messina et al., 2016). Spatially explicit information on where the disease

407 occurs, covering both asymptomatic and symptomatic infections and derived from representative,

408 standardized surveys would be most desirable. In practice, the location of reporting and the 409 generally not reported site of infection can also lead to misidentification of ecological conditions

410 favouring disease occurrence (Allen et al. 2017). In addition, regional differences in health care,

411 i.e. countryside vs. city, can lead to a sampling bias, with fewer cases reported from the former.

412 Another limitation in the data used for the risk model might be that the number of reported Zika

413 cases is likely underestimated as the majority of ZIKV infections causes only mild, flu-like

414 symptoms (Messina et al., 2016), which are often unrecognised and not reported. Occurrence data

415 affected by sampling bias is possibly the biggest weakness of any kind of modelling approach.

416 Accounting for sampling bias in the modelling approach requires the bias being captured, e.g.

417 using information on how and where other diseases are generally reported in South America.

418 Without this information, a meaningful estimation of bias is not possible.

419 The high variability in space and time during outbreaks is typical for emerging infectious diseases

420 like Zika. The ENM approach does not account for the temporal dynamics of the virus'

421 occurrences. For such temporal dynamics process-based, mathematical epidemic models would be

422 more appropriate, however, these models require many parameters, which are difficult to estimate. 
423 The strength of the correlative approaches is that they are relatively easy to perform; however,

424 limitations should always be acknowledged and diminished. Thus, whether introductions of ZIKV

425 will result in its establishment and endemic disease outbreak depends on many other factors than

426 those considered here, e.g. vector abundance, population immunity, access to health services, but

427 also random chance (Lessler et al., 2016). Clearly, there is a need to continue monitoring and

428 surveillance of all components associated to ZIKA, especially in those countries and regions where

429 the transmission risk is high.

430

\section{Conclusion}

432 Worldwide, evidence of autochthonous mosquito-borne ZIKV transmission has been reported 433 from 87 countries and territories (as of July 2019, WHO 2019) and there is still a risk for further 434 spread of ZIKV (WHO, 2019). In response to the emergence and global spread of ZIKV infections 435 and associated complications, public health systems need to be strengthened and should include 436 epidemiological surveillance. Disease biogeography is currently a promising field to complement 437 epidemiology. Applying the concepts and tools from ENM to disease biogeography and 438 epidemiology will provide biologically sound and analytically robust descriptive and predictive 439 analyses of disease distributions, help designing evidence-based control strategies, and allow 440 comprehensive identification of potential transmission areas to allocate resources for surveillance

441 (Escobar 2017, Johnson et al. 2019). 


\section{Figure legends}

444 Figure 1: Graphical abstract of the considered approach modified after Messina et al, 2015. The 445 ZIKV transmission risk is modelled based on seven predictor variables: modelled habitat 446 suitability for the two main vector species (a1) Aedes aegypti and (a2) Aedes albopictus, (b) 447 temperature of warmest quarter, (c) Zika evidence consensus map, (d) Dengue evidence consensus 448 map, (e) population density and (f) gross domestic product.

Figure 2: Scoring system to calculate the evidence consensus (EC) values on a country level.

451

452

453

454

455 456 457

458 459

462

463 464 465
Modified version after Brady et al. (2012). Information provided by the WHO, CDC, PAHO and GIDEON. The classification was relative to the regional values, which in turn were derived from the national class according to WHO and Gideon.

Figure 3: Modelled habitat suitability of the two main vector species a) Aedes aegypti and b) Aedes albopictus. Warmer colours indicate higher modelled habitat suitability.

Figure 4: Evidence consensus map for Zika on a regional scale. Evidence consensus values between 0 (dark green) and 12 (red) according to the scoring system described in Figure 2 for Zika in South and Central America.

Figure 5: Modelled ZIKV transmission risk in South and Central America based on Maxent cloglog output: dark green: $0-0.03$, light green: $0.03-0.16$, yellow: $0.16-0.30$, orange: $0.30-$ 0.62 , red: $0.62-1$. Classified by percentiles.

Peer) reviewing PDF | (2019:04:37005:2:0:NEW 27 Aug 2019) 


\section{List of acronyms}

467 Ae. - Aedes

468 CDC - Centers for Disease Control and Prevention

469 cf. - lat. confer

470 ECM - evidence consensus map

471 ENM - ecological niche mode

472 ESA - European Space Agency

473 esri - Environmental Systems Research Institute

474 GDP - gross domestic product

475 Gideon - Global Infectious Disease and Epidemiology Network

476 GIS - geographical information system

477 LCCS - United Nations Land Cover Classification Systems

478 PAHO - Pan American Health Organization

479 PCA - principal component analysis

480 WHO - world health organization

481 ZIKV - Zika virus

482 


\section{Acknowledgements}

484 The present study was supported by the Uniscientia Stiftung. The present study was also

485 supported by the LOEWE-Centre TBG funded by the Hessen State Ministry of Higher

486 Education, Research, and the Arts (HMWK). 
487

488

489

490

491

492

493

494

495

496

497

498

499

500

501

502

503

504

505

506

507

\section{References}

Aiello-Lammens ME, Boria RA, Radosavljevic A, Vilela B, Anderson RP. 2015. spThin: an R package for spatial thinning of species occurrence records for use in ecological niche models. Ecography. 38(5): 541-545, 2015 DOI: https://doi.org/10.1111/ecog.01132

Allen T, Murray KA, Zambrana-Torrelio C, Morse SS, Rondinini C, Di Marco M, Breit N, Olival KJ, Daszak P. 2017. Global hotspots and correlates of emerging zoonotic diseases. Nature Communications. 8: 1124 DOI: 10.1038/s41467-017-00923-8

Anderson RP. 2013. A framework for using niche models to estimate impacts of climate change on species distributions. Annals of the New York Academy of Science. Issue: Climate change and species interactions: Ways forward. ISSN 0077-8923

Anderson RP \& Gonzalez I. 2011. Species-specific tuning increases robustness to sampling bias in models of species distributions: an implementation with MAXENT. Ecological Modelling. 222(15): 2796-2811. DOI: 10.1016/j.ecolmodel.2011.04.011

Arino O, Ramos Peres JJ, Kalogirou V, Bontemps S, Defourny P, van Bogaert E. 2012. Global Land Cover Map for 2009 (GlobCover 2009). With assistance of (C) European Space Agency \& Université catholique de Louvain.

Balogun EO, Nok AJ, Kita K .2016. Global warming and the possible globalization of vectorborne diseases: a call for increased awareness and action. Tropical Medicine and Health 44:38. DOI: $10.1186 / \mathrm{s} 41182-016-0039-0$.

Baak-Baak CM, Moo-Llanes DA, Cigarroa-Toledo N, Puerto FI, Machain-Williams C, Reyes-Solis G, Nakazawa YJ, Ulloa-Garcia A, Garcia-Rejon JE. 2017. Ecological niche model 
508 for predicting distribution of disease-vector mosquitoes in Yucatán State, México. Journal of 509 Medical Entomology 54(4): 854-861. DOI: 10.1093/jme/tjw243.

510 Bhatt S, Gething PW, Brady OJ, Messina JP, Farlow AW, Moyes CL, Drake JM, Brownstein

511 JS, Hoen AG, Sankoh O, Myers MF, George DB, Jaenisch T, Wint GR, Simmons CP, Scott

512 TW, Farrar JJ, Hay SI... 2013. The global distribution and burden of dengue. Nature 513 496(7446):504-507. DOI: 10.1038/nature12060.

514

515

516

517

518

519

520

521

522

523

524

525

526

527

528

Brady OJ, Gething PW, Bhatt S, Messina JP, Brownstein JS, Hoen AG, Moyes CL, Farlow

AW, Scott TW, Hay SI. 2012. Refining the global spatial limits of dengue virus transmission by evidence-based consensus. PLoS Neglected Tropical Diseases 6(8):e1760. DOI: 10.1371/journal.pntd.0001760.

Brand SP, Keeling MJ. 2017. The impact of temperature changes on vector-borne disease transmission: Culicoides midges and bluetongue virus. Journal of the Royal Society, Interface. 14(128):20160481. DOI: 10.1098/rsif.2016.0481.

Caminade C, Turner J, Metelmann S, Hesson JC, Blagrove MSC, Solomon T, Morse AP, Baylis M. 2017. Global risk model for vector-borne transmission of Zika virus reveals the role of El Niño 2015. Proceedings of the National Academy of Sciences of the United States of America 114(1):119-124. DOI: 10.1073/pnas.1614303114.

Carlson CJ, Dougherty ER, Getz W. 2016. An ecological assessment of the pandemic threat of Zika virus. PLoS Neglected Tropical Diseases 10(8):e0004968. DOI: 10.1371/journal.pntd.0004968.

Cohen J. 2017. Dengue may bring out the worst in Zika. Science. DOI: 10.1126/science.aal0982. 
529 Cunze S, Tackenberg O. 2015. Decomposition of the maximum entropy niche function - A step

530 beyond modelling species distribution. Environmental Modelling \& Software 72:250-260. DOI:

$531 \quad 10.1016 /$ j.envsoft.2015.05.004.

532 de Brito CAA, de Brito CCM, Oliveira AC, Rocha M, Atanásio C, Asfora C, Matos JD, Lima

533 AS, Albuquerque MFM. 2016. Zika in Pernambuco: Rewriting the first outbreak. Revista da

534 Sociedade Brasileira de Medicina Tropical, 49(5): 553-558. DOI: 10.1590/0037-8682-0245-2016

535

536

537

538

539

540

541

542

543

544

545

546

547

548

549

550

\section{Dejnirattisai W, Supasa P, Wongwiwat W, Rouvinski A, Barba-Spaeth G, Duangchinda T,} Sakuntabhai A, Cao-Lormeau V, Malasit P, Rey FA, Mongkolsapaya J, Screaton GR. 2016.

Dengue virus sero-cross-reactivity drives antibody-dependent enhancement of infection with Zika virus. Nature Immunology 17(9):1102-1108. DOI: 10.1038/ni.3515.

Dupont-Rouzeyrol M, O'Connor O, Calvez E, Daurès M, John M, Grangeon JP, Gourinat AC. 2015. Co-infection with Zika and dengue viruses in two patients New Caledonia 2014. Emerging Infectious Diseases 21(2):381-382. DOI: 10.3201/eid2102.141553.

Elith J, Graham CH, Anderson RP, Dudík M, Ferrier S, Guisan A, Hijmans RJ, Leathwick FHJR, Lehmann A, Li J, Lohmann LG, Loiselle BA, Manion G, Nakamura CMM, Nakazawa Y, McC. Overton J, Peterson AT, Phillips SJ, Richardson K, Scachetti-Pereira R, Schapire RE, Soberón J, Williams S, Wisz MS, Zimmermann NE. 2006. Novel methods improve prediction of species' distributions from occurrence data. Ecography 29: 129-151.

ESA GlobeCover 2009 Project (2010) Data on Land cover of Central and South America (GlobCover2009). Available at:

https://databasin.org/datasets/3f40feca13f649438f3d6b6162ff4709, accessed via ArcGIS online (October 2017) 
551 Escobar LE, Craft ME. 2016. Advances and limitations of disease biogeography using ecological

552 niche modeling. Frontiers in Microbiology 7:1174. DOI: 10.3389/fmicb.2016.01174.

553 Escobar LE, Qiao H, Lee C, Phelps NBD. 2017. Novel methods in disease biogeography: A case

554 study with Heterosporosis. Frontiers in Veterinary Science 4:105. DOI:

555 10.3389/fvets.2017.00105;4:105. Published 2017 Jul 17. doi:10.3389/fvets.2017.00105

556 Fick SE, Hijmans RJ. 2017. Worldclim 2: New 1-km spatial resolution climate surfaces for global 557 land areas. International Journal of Climatology.

558 Funk S, Kucharski AJ, Camacho A, Eggo RM, Yakob L, Murray LM, Edmunds WJ. 2016.

559 Comparative analysis of Dengue and Zika outbreaks reveals differences by setting and virus. PLoS

560 Neglected Tropical Diseases.10(12):e0005173. doi:10.1371/journal.pntd.0005173

561 Gardner LM, Bóta A, Gangavarapu K, Kraemer MUG, Grubaugh ND. 2018. Inferring the 562 risk factors behind the geographical spread and transmission of Zika in the Americas. PLoS 563 Neglected Tropical Diseases 12(1):e0006194. DOI: 10.1371/journal.pntd.0006194.

Gordon A, Gresh L, Ojeda S, Katzelnick LC, Sanchez N, Mercado JC, Chowell G, Lopez B, 565

Elizondo D, Coloma J, Burger-Calderon R, Kuan G, Balmaseda A, Harris E. 2019. Prior 566 dengue virus infection and risk of Zika: A pediatric cohort in Nicaragua. PLoS Medicine 567 16(1):e1002726. Doi: 10.1371/journal.pmed.1002726.

568

569

570

571
Heitmann A, Jansen S, Lühken R, Leggewie M, Badusche M, Pluskota B, Becker N, Vapalahti O, Schmidt-Chanasit J, Tannich E. 2017. Experimental transmission of Zika virus by mosquitoes from central Europe. Eurosurveillance 22(2):30437. DOI: 10.2807/15607917.ES.2017.22.2.30437. 
572 Huang X, Yue Y, LD, Zhao Y, Qiu L, Chen J, Pan Y, Xi J, Wang X, Sun Q, Li Q. 2016.

573 Antibody-dependent enhancement of dengue virus infection inhibits RLR-mediated Type-I IFN-

574 independent signalling through upregulation of cellular autophagy. Scientific Reports 6:22303.

575 DOI: $10.1038 /$ srep22303.

576 Johnson EE, Escobar LE, Zambrana-Torrelio C. 2019. An ecological framework for modeling

577 the geography of disease transmission. Trends in Ecology \& Evolution. 2521. DOI:

578 https://doi.org/10.1016/j.tree.2019.03.004

579 Kraemer MUG, Sinka ME, Duda KA, Mylne A, Shearer FM, Brady OJ, Messina JP, Barker

580

CM, Moore CG, Carvalho RG, Coelho GE, Van Bortel W, Hendrickx G, Schaffner F, Wint

581

GRW, Elyazar IRF, Teng H, Hay SI. 2015a. Data from: The global compendium of Aedes

582 aegypti and Ae. albopictus occurrence.

Kraemer MUG, Sinka ME, Duda KA, Mylne A, Shearer FM, Brady OJ, Messina JP, Barker

GRW, Elyazar IRF, Teng H, Hay SI. 2015b. The global compendium of Aedes aegypti and Ae. albopictus occurrence. Scientific Data 2:150035. DOI: 10.1038/sdata.2015.35.

Krauer F, Riesen M, Reveiz L, Oladapo OT, Martínez-Vega R, Porgo TV. Haefliger A,

Broutet NJ, Low N. 2017. Zika Virus Infection as a Cause of Congenital Brain Abnormalities 589 and Guillain-Barré Syndrome: Systematic Review. PLoS Medicine 14(1):e1002203. DOI: 590 10.1371/journal.pmed.1002203.

Lessler J, Chaisson LH, Kucirka LM, Bi Q, Grantz K, Salje H, Carcelen AC. 2016. Assessing 592 the global threat from Zika virus. Science 353(6300):aaf8160. DOI: $10.1126 /$ science.aaf8160. 
593 Leutner B, Horning N. 2017. RStoolbox: Tools for Remote Sensing Data Analysis. R Package

594 Version 0.1.10. Available online at https://CRAN.R-project.org/package=RStoolbox.

595 Lo D, Park B. 2018. Modeling the spread of the Zika virus using topological data analysis. PloS

596 One 13(2):e0192120. DOI: 10.1371/journal.pone.0192120.

597 Lounibos LP, Juliano SA. 2018. Where vectors collide. The importance of mechanisms shaping 598 the realized niche for modeling ranges of invasive Aedes mosquitoes. Biological Invasions 599 20(8):1913-1929. DOI: 10.1007/s10530-018-1674-7.

Medlock JM, Leach SA. 2015. Effect of climate change on vector-borne disease risk in the UK.

The Lancet Infectious Diseases 15(6):721-730. DOI: 10.1016/S1473-3099(15)70091-5.

602

603

604

605

606

607

608

609

610

Messina J, Shearer F. 2016. Global compendium of human Zika virus occurrence. Retrieved: 08:48 Dec 062017 (GMT). figshare. https://doi.org/10.6084/m9.figshare.2573629.v1

Messina JP, Brady OJ, Pigott DM, Golding N, Kraemer MUG, Scott TW, Wint GRW, Smith

DL, Hay SI. 2015. The many projected futures of dengue. Nature Reviews. Microbiology 13(4):230-239. DOI: $10.1038 /$ nrmicro3430.

Messina JP, Kraemer MUG, Brady OJ, Pigott DM, Shearer FM, Weiss DJ, Golding N, Ruktanonchai CW, Gething PW, Cohn E, Brownstein JS, Khan K, Tatem AJ, Jaenisch T, Murray CJL, Marinho F, Scott TW, Hay SI. 2016. Mapping global environmental suitability for Zika virus. eLife 5. DOI: 10.7554/eLife.15272.

Mordecai EA, Cohen JM, Evans, MV, Gudapati P, Johnson LR, Lippi CA, Miazgowicz K, Murdock CC, Rohr JR, Ryan SJ, Savage V, Shocket MS, Ibarra AS, Thomas MB, Weikel DP. 2017. Detecting the impact of temperature on transmission of Zika dengue and chikungunya 
614 using mechanistic models. PLoS Neglected Tropical Diseases 11(4):e0005568. DOI: 615 10.1371/journal.pntd.0005568.

616 Phillips SJ, Anderson RP, Dudík M, Schapire RE, Blair M. 2017. Opening the black box: an 617 open-source release of Maxent. Ecography 40: 887-893. DOI: 10.1111/ecog.03049

618 Phillips SJ, Dudík M, Schapire RE. [Software] Maxent software for modelling species niches 619 and distributions (Version 3.4.1).

620 Pigott DM, Golding N, Mylne A, Huang Z, Weiss DJ, Brady OJ, Kraemer MUG, Hay SI. 621 2015. Mapping the zoonotic niche of Marburg virus disease in Africa. Transactions of the Royal 622 Society of Tropical Medicine and Hygiene 109(6): 66-378. DOI: 10.1093/trstmh/trv024

623 R Core Team. 2014. R: A Language and Environment for Statistical Computing. R Foundation 624 for Statistical Computing Vienna Austria.

Radosavljevic A \& Anderson RP. 2014. Making better Maxent models of species distributions: 626 complexity, overfitting and evaluation. Journal of Biogeography. 41(4): 629-643. DOI: 627 https://doi.org/10.1111/jbi.12227

Rodriguez-Barraquer I, Costa F, Nascimento EJM, Junior NN, Castanha PMS, Sacramento Azar SR, Rossi SL, Vasilakis N, Weaver SC, Ribeiro GS, Balmaseda A, Harris E, Nogueira

632 preexisting dengue immunity on Zika virus emergence in a dengue endemic region. Science, 633 363(6427): 607-610. 
634 Romeo-Aznar V, Paul R, Telle O, Pascual M. 2018. Mosquito-borne transmission in urban 635 landscapes: the missing link between vector abundance and human density. Proceedings of the 636 Royal Society. Biological sciences 285(1884). DOI: 10.1098/rspb.2018.0826.

637 Samuel GH, Adelman ZN, Myles KM. 2016. Temperature-dependent effects on the replication 638 and transmission of arthropod-borne viruses in their insect hosts. Current Opinion in Insect 639 Science 16:108-113. DOI: 10.1016/j.cois.2016.06.005.

640 Samy AM, Campbell LP, Peterson AT. 2014. Leishmaniasis transmission: distribution and 641 coarse-resolution ecology of two vectors and two parasites in Egypt. Revista da Sociedade 642 Brasileira de Medicina Tropical. 47(1):57-62. DOI: 10.1590/0037-8682-0189-2013.

643 Samy AM, Thomas SM, Wahed AAE, Cohoon KP, Peterson AT. 2016. Mapping the global 644 geographic potential of Zika virus spread. Memórias do Instituto Oswaldo Cruz 111(9):559-560. 645 DOI: 10.1590/0074-02760160149.

646 Suparit P, Wiratsudakul A, Modchang C. 2018. A mathematical model for Zika virus 647 transmission dynamics with a time-dependent mosquito biting rate. Theoretical Biology and 648 Medical Modelling 15(11). DOI: 10.1186/s12976-018-0083-z

649 Tesla B, Demakovsky LR, Mordecai EA, Ryan SJ, Bonds MH, Ngonghala CN, Brindley MA, 650 Murdock CC. 2018. Temperature drives Zika virus transmission: evidence from empirical and 651 mathematical models. Proceedings of the Royal Society. Biological sciences 285(1884). DOI: $65210.1098 /$ rspb.2018.0795.

653 Tsai CH, Chen TH, Lin C, Shu PY, Su CL, Teng HJ. 2017. The impact of temperature and 654 Wolbachia infection on vector competence of potential dengue vectors Aedes aegypti and Aedes 
655 albopictus in the transmission of dengue virus serotype 1 in southern Taiwan. Parasites \& Vectors.

656 10(1):551. DOI: 10.1186/s13071-017-2493-x.

657 WHO 2016. Zika situation report. Zika virus Microcephaly and Guillain-Barré syndrome—31

658 March 2016. Situation Report 2016. Available online at http://www.who.int/emergencies/zika-

659 virus/situation-report/31-march-2016/en/ checked on 7/16/2018.

660 WHO 2019. Zika epidemiology update. July 2019. Situation Report 2016. Available online at 661 https://www.who.int/emergencies/diseases/zika/zika-epidemiology-update-july-2019.pdf?ua=1 662 checked on 7/11/2019.

663 Wiwanitkit S, Wiwanitkit V. 2016. Predicted pattern of Zika virus infection distribution with 664 reference to rainfall in Thailand. Asian Pacific Journal of Tropical Medicine 9(7):719-720. DOI: 665 10.1016/j.apjtm.2016.05.014.

666 Županc AT, Petrovec M. 2016. Zika: An old virus with a new face. Zdravstveno varstvo 667 55(4):228-230. DOI: 10.1515/sjph-2016-0031. 
Figure 1

Graphical abstract of the considered approach modified after Messina et al, 2015.

The ZIKV transmission risk is modelled based on the following seven predictor variables: modelled habitat suitability for the two main vector species (a1) Aedes aegypti and (a2) Aedes albopictus, (b) temperature of warmest quarter, (c) Zika evidence consensus map, (d) Dengue evidence consensus map, (e) population density and (f) gross domestic product.

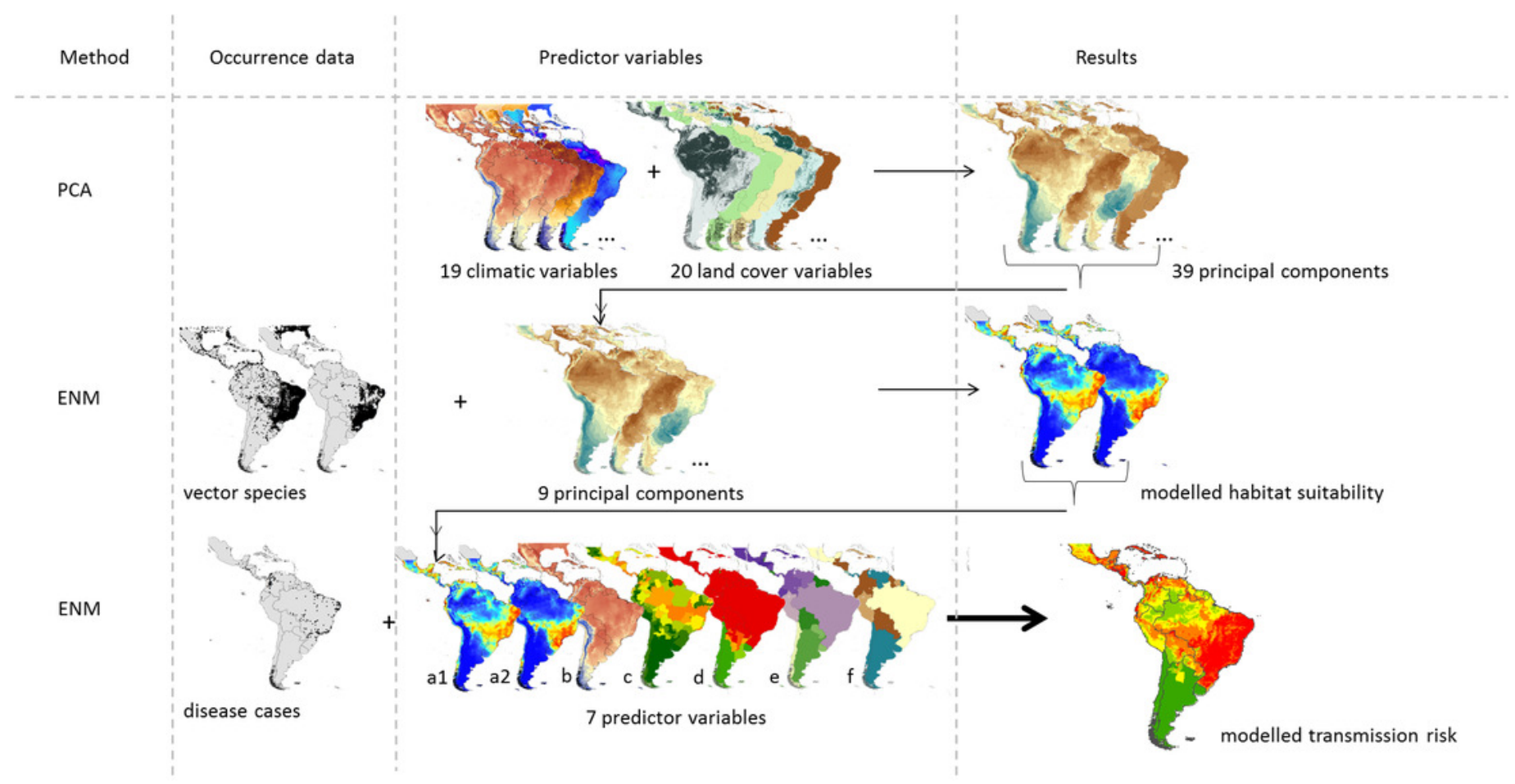


Figure 2

Scoring system to calculate the evidence consensus (EC) values on a country level, modified version after Brady et al. (2012).

Information provided by the WHO, CDC, PAHO and GIDEON. The classification was relative to the regional values, which in turn were derived from the national class according to WHO and Gideon.

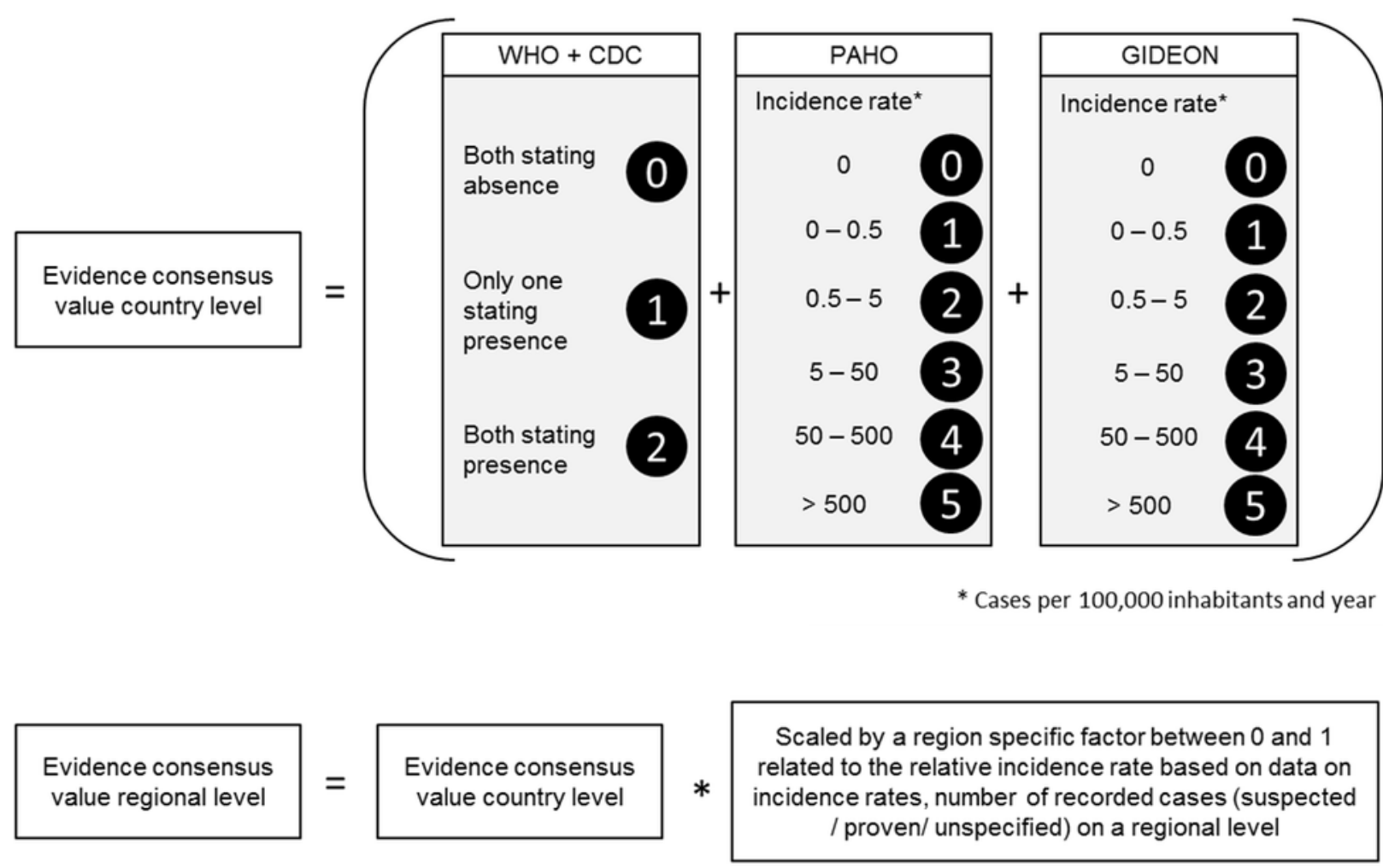


Figure 3

Modelled habitat suitability of the two main vector species a) Aedes aegypti and b) Aedes albopictus.

Warmer colours indicate higher modelled habitat suitability.

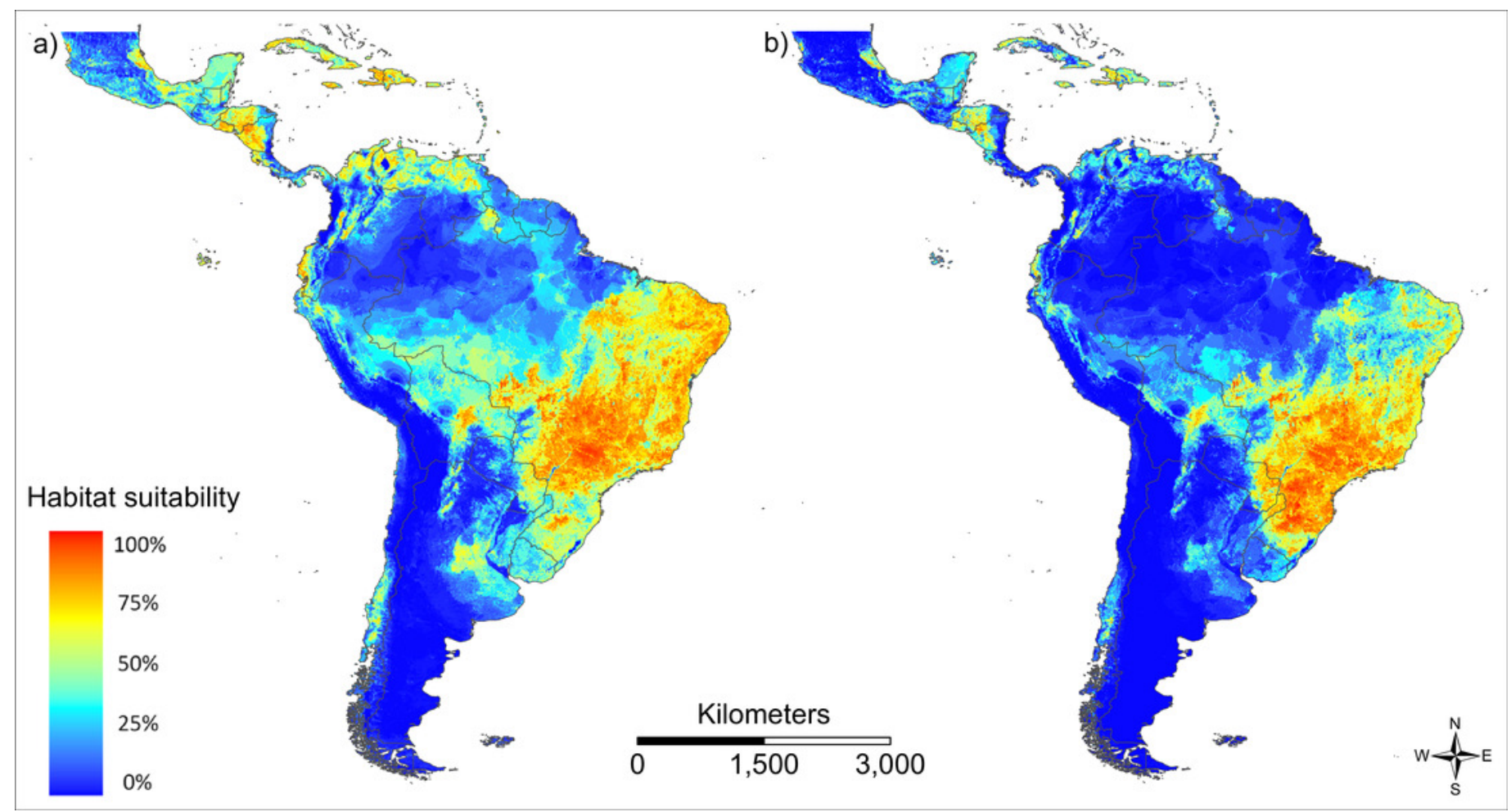


Figure 4

Evidence consensus map for Zika on a regional scale.

Evidence consensus values between 0 (dark green) and 12 (red) according to the scoring system described in Figure 2 for Zika in South and Central America. 


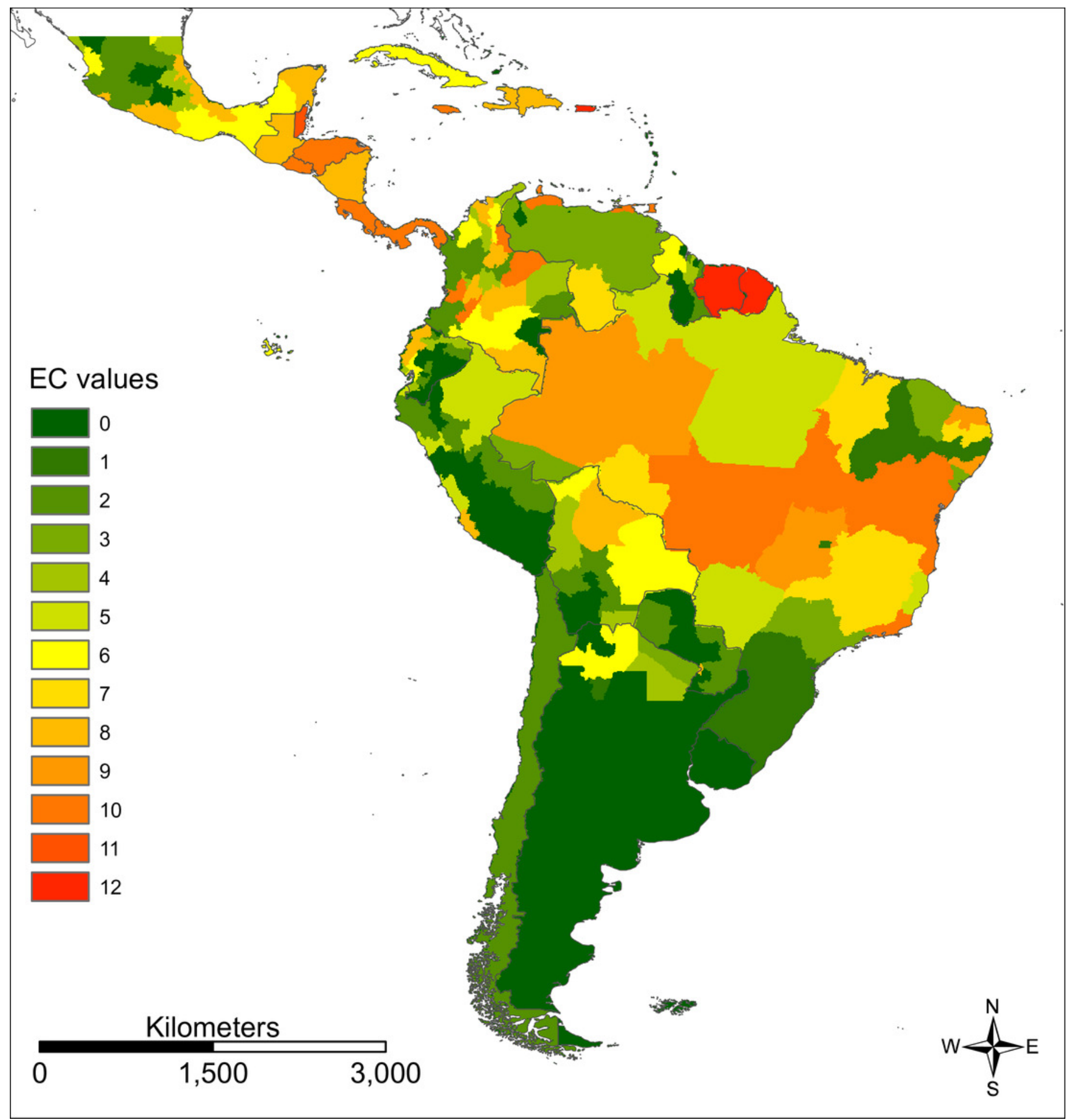


Figure 5

Modelled ZIKV transmission risk in South and Central America based on Maxent cloglog output.

dark green: $0-0.03$, light green: $0.03-0.16$, yellow: $0.16-0.30$, orange: $0.30-0.62$, red: $0.62-1$. Classified by percentiles. 


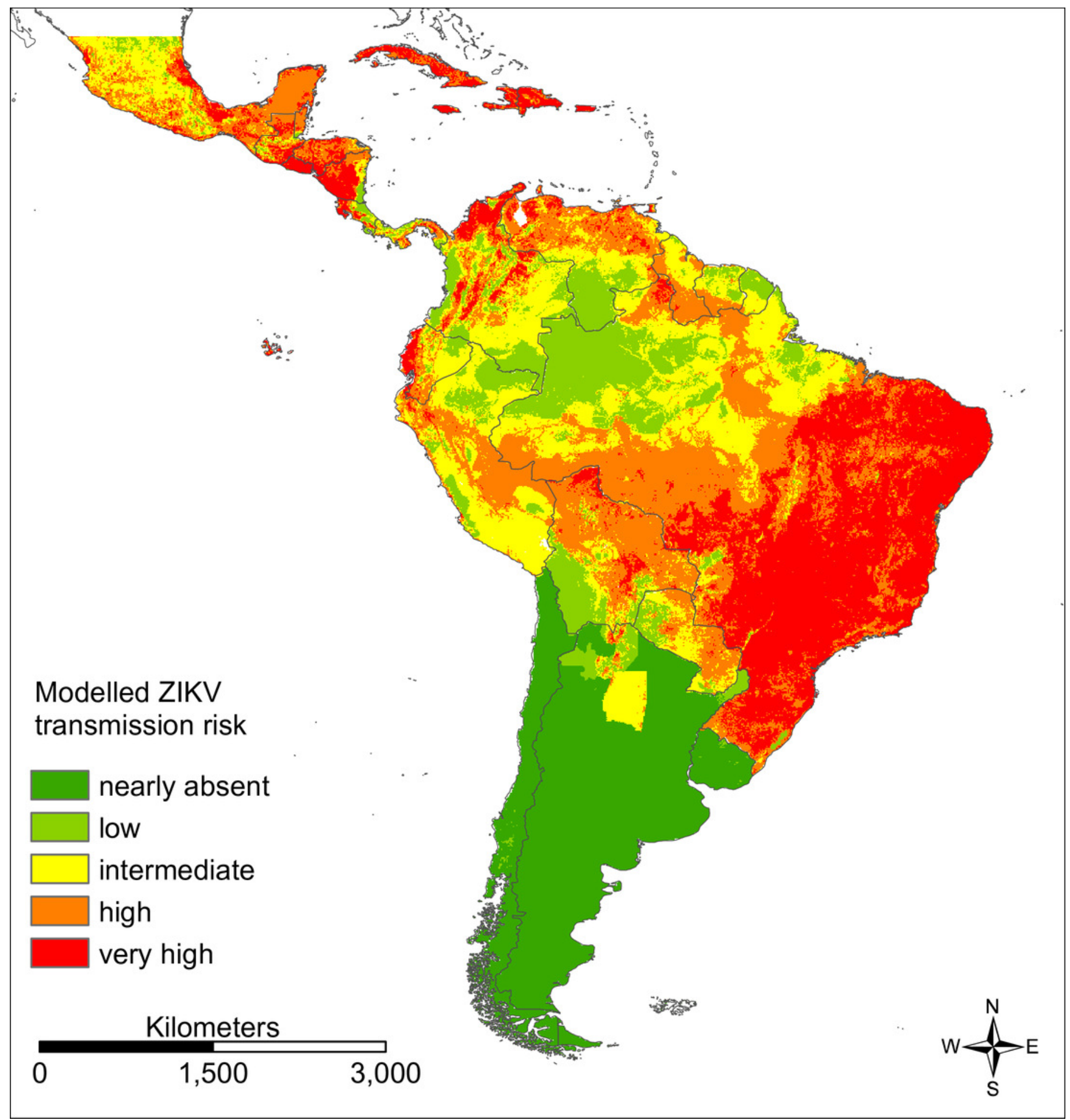

